\title{
Influence of microscopic many-body scattering on the performance of ultrashort pulsed and CW multi- wavelength VECSEL lasing
}

I. Kilen, J. V. Moloney, J. Hader, S. W. Koch

I. Kilen, J. V. Moloney, J. Hader, S. W. Koch, "Influence of microscopic manybody scattering on the performance of ultrashort pulsed and CW multiwavelength VECSEL lasing," Proc. SPIE 10515, Vertical External Cavity Surface Emitting Lasers (VECSELs) VIII, 1051504 (15 February 2018); doi: 10.1117/12.2291407

SPIE. Event: SPIE LASE, 2018, San Francisco, California, United States 


\title{
Influence of Microscopic Many-Body Scattering on the performance of Ultrashort Pulsed and CW Multi-wavelength VECSEL Lasing
}

\author{
I. Kilen ${ }^{a}$, J. V. Moloney ${ }^{a, b, c}, \mathrm{~J}_{\text {. Hader }}^{b}$, and S. W. Koch ${ }^{b, d}$. \\ ${ }^{a}$ Program in Applied Mathematics, University of Arizona, Tucson, Arizona 85721, USA; \\ ${ }^{b}$ College of Optical Sciences, The University of Arizona, \\ 1630 East University Boulevard, Tucson, Arizona 85721, USA; \\ ${ }^{c}$ Department of Mathematics, University of Arizona, \\ 617 N. Santa Rita, Tucson, AZ 85721, USA \\ ${ }^{d}$ Department of Physics and Material Sciences Center, Philipps-Universität Marburg, \\ Renthof 5, 35032 Marburg, Germany;
}

\begin{abstract}
The influence of microscopic non-equilibrium dynamics on vertical external-cavity surface-emitting lasers (VECSELs) is investigated through a systematic numerical study of single- and dual-wavelength operation. In singlewavelength operation the microscopic dynamics can be adiabatically eliminated, however in dual-wavelength operation the microscopic dynamics varies with the spectral location of the modes. The optically active quantum wells (QWs) are modeled microscopically using the Semiconductor Bloch equations while the CW laser field is simulated using Maxwell's equations. Higher order correlation terms, such as carrier scattering and polarization dephasing, are treated on the level of second Born-Markov or as effective rates. Results are presented on the modeling, stability, and non-equilibrium effects in dual-wavelength operation.
\end{abstract}

Keywords: VECSELs, semiconductor laser, mode-locking, multi-wavelength, simulation, semiconductor Bloch equations, RPG, GaAs

\section{INTRODUCTION}

Vertical external-cavity surface-emitting lasers have been used in many configurations such as for mode-locked pulse operation and single- or multi-wavelength operation. ${ }^{1-17}$ In the pursuit of high output power there have been reports of $23 \mathrm{~W}$ single-wavelength operation and $106 \mathrm{~W}$ multi-wavelength operation. ${ }^{6,7,14}$ Mode-locked pulses have been generated using multiple different saturable absorbers: semiconductor external saturable absorber mirrors (SESAMs), ${ }^{1,2,4,8,9}$ graphene and carbon nanotube saturable absorbers (GSAM) ${ }^{10-12}$ and integrated quantum well (QW) or quantum dot saturable absorbers. ${ }^{10}$ To date, the shortest stable mode-locked pulse inside a VECSEL has a temporal width of $107 \mathrm{fs} .{ }^{13,16}$ In addition, a pulse trail of $60 \mathrm{fs}$ pulses has been found inside a picosecond envelope. ${ }^{3}$

A numerical model for a VECSEL has to include the coupling of the field propagation to the microscopic QW dynamics. A possibility is to derive rate equations, where the microscopic QW polarization dynamics is adiabatically eliminated by assuming that carriers are in equilibrium Fermi distributions. ${ }^{18,19}$ Thus reducing the nonlinear QW carrier dynamics to many fit parameters that are experimentally approximated e.g. the amplitude of the QW gain that is assumed to have a parabolic shape. ${ }^{18}$ However, a VECSEL configured for high-power or ultrashort pulse generation will naturally generate intracavity fields that drive the QW carriers far from equilibrium, which will result in significant gain deformation and a departure from equilibrium dynamics. In

Further author information:

I. K.: Electronic mail: ikilen@math.arizona.edu

Vertical External Cavity Surface Emitting Lasers (VECSELs) VIII, edited by

Juan L. Chilla, Proc. of SPIE Vol. 10515, 1051504 - C) 2018 SPIE

CCC code: $0277-786 \mathrm{X} / 18 / \$ 18 \cdot$ doi: $10.1117 / 12.2291407$

Proc. of SPIE Vol. 10515 1051504-1 
these situations, a quantitative microscopic model is required in order to study the influence of the microscopic dynamics on the cavity field.

In this paper, we model a VECSEL using the Maxwell semiconductor Bloch equations (MSBE) where Maxwell's equation propagates the electric field and the semiconductor Bloch equations (SBE) model the light interaction with QWs. ${ }^{20}$ When appropriate, we extract effective rates for higher order correlation contributions such as for polarization dephasing, carrier scattering, and carrier relaxation. The MSBE has been used in the past to study mode-locked VECSELs and has given deep insight into the role of microscopic dynamics. ${ }^{21-30}$ In this paper, we will give an overview of the role of microscopic dynamics on the mode-locked pulse and multiwavelength operation of VECSELs.

\section{THEORETICAL BACKGROUND}

In a VECSEL, the electromagnetic field propagation is governed by Maxwell's equations while the interaction with the optically active QWs follows the SBE. In order to provide a quantitative model, we will solve the coupled MSBE equations for the entire VECSEL. However, this is a system of nonlinear equations, with thousands of variables, that have to be solved iteratively on a sub-femtosecond timescale to fully capture the microscopic dynamics. A numerical solution to these equations is a significant computational challenge and we will make some simplifications in order to provide some insight into the role of the microscopic dynamics. First, the propagation of the field is considered perpendicular to the QW planes along the z-axsis. In this case, we can reduce Maxwell's equation for the electric field $E(z, t)$ to a $1 \mathrm{D}$ wave equation where the QWs interact with the electromagnetic field through the macroscopic polarization $P(z, t)$

$$
\left[\frac{\partial^{2}}{\partial z^{2}}-\frac{n(z)^{2}}{c_{0}^{2}} \frac{\partial^{2}}{\partial t^{2}}\right] E(z, t)=\mu_{0} \frac{\partial^{2}}{\partial t^{2}} P(z, t),
$$

where $c_{0}$ is the speed of light in vacuum, $n$ is the background refractive index of a material, and $\mu_{0}$ is the vacuum permeability. We will assume a linear simulation domain where the gain chip is placed on the left with an output coupling mirror on the right. The external cavity is an air gap that is placed between the optically active components. The gain chip is based on a realistic design that incorporates a distributed Bragg reflector (DBR), a series of inverted QWs inside a GaAs barrier, a cap layer, and dispersion compensating coating. As the electromagnetic field propagates throughout the simulation domain the field will experience reflection/transmission from each material interface and amplification (or absorption) from the optically active QWs.

The SBE will model the microscopic many-body dynamics in each QW. ${ }^{20}$ Here, the time dependent carrier occupation numbers $\left(n_{\lambda(\nu), \mathbf{k}}^{\mathrm{e}(\mathrm{h})}\right)$ and microscopic polarizations $\left(p_{\lambda, \nu, \mathbf{k}}\right)$ are simulated directly for electrons (holes) in conduction (valence) bands $\lambda(\nu)$. An electric field will directly influence the carrier occupation numbers and microscopic polarizations that follows:

$$
\begin{aligned}
\frac{\partial}{\partial t} p_{\lambda, \nu, \mathbf{k}} & =-\frac{\mathrm{i}}{\hbar} \sum_{\lambda_{1}, \nu_{1}}\left(e_{\lambda, \lambda_{1}, \mathbf{k}}^{\mathrm{e}} \delta_{\nu, \nu_{1}}+e_{\nu, \nu_{1}, \mathbf{k}}^{\mathrm{h}} \delta_{\lambda, \lambda_{1}}\right) p_{\lambda_{1}, \nu_{1}, \mathbf{k}}-\mathrm{i}\left(n_{\lambda, \mathbf{k}}^{\mathrm{e}}+n_{\nu, \mathbf{k}}^{\mathrm{h}}-1\right) \Omega_{\lambda, \nu, \mathbf{k}}+\Lambda_{\lambda, \nu, \mathrm{spont}}^{p}+\left.\frac{\partial}{\partial t} p_{\lambda, \nu, \mathbf{k}}\right|_{\text {corr }}, \\
\frac{\partial}{\partial t} n_{\lambda(\nu), \mathbf{k}}^{\mathrm{e}(\mathrm{h})} & =-2 \operatorname{Im}\left(\Omega_{\lambda, \nu, \mathbf{k}}\left(p_{\lambda, \nu, \mathbf{k}}\right)^{*}\right)+\Gamma_{\lambda(\nu), \mathrm{scatt}}^{\mathrm{e}(\mathrm{h})}+\Lambda_{\lambda, \nu, \mathrm{spont}}^{n}+\left.\frac{\partial}{\partial t} n_{\lambda(\nu), \mathbf{k}}^{e(h)}\right|_{\text {corr }} ^{e},
\end{aligned}
$$

where the carrier in-plane momentum is denoted by $\mathbf{k}$ and the macroscopic polarization is calculated using $P(z, t)=\sum_{\mathbf{k}} d_{\mathbf{k}}^{\lambda, \nu} p_{\lambda, \nu, \mathbf{k}}$. The Hartree-Fock renormalized single-particle energies $\left(e_{\lambda, \lambda_{1}, \mathbf{k}}^{\mathrm{e}}\right.$ and $\left.e_{\nu, \nu_{1}, \mathbf{k}}^{\mathrm{h}}\right)$ and the effective Rabi frequency $\left(\Omega_{\lambda, \nu, \mathbf{k}}\right)$ are given by:

$$
\begin{aligned}
& e_{\lambda, \lambda_{1}, \mathbf{k}}^{\mathrm{e}}=\epsilon_{\lambda, \mathbf{k}}^{\mathrm{e}} \delta_{\lambda, \lambda_{1}}-\sum_{\lambda_{2}, \mathbf{q}} V_{|\mathbf{k}-\mathbf{q}|}^{\lambda, \lambda_{2}, \lambda_{1}, \lambda_{2}} n_{\lambda_{2}, \mathbf{q}}^{\mathrm{e}} \\
& e_{\nu, \nu_{1}, \mathbf{k}}^{\mathrm{h}}=\epsilon_{\nu, \mathbf{k}}^{\mathrm{h}} \delta_{\nu, \nu_{1}}-\sum_{\nu_{2}, \mathbf{q}} V_{|\mathbf{k}-\mathbf{q}|}^{\nu, \nu_{2}, \nu_{1}, \nu_{2}} n_{\nu_{2}, \mathbf{q}}^{\mathrm{h}}
\end{aligned}
$$


and

$$
\Omega_{\lambda, \nu, \mathbf{k}}=\omega_{\mathrm{R}}+\frac{1}{\hbar} \sum_{\lambda_{1}, \nu_{1}, \mathbf{q} \neq \mathbf{k}} V_{|\mathbf{k}-\mathbf{q}|}^{\lambda, \nu_{1}, \nu, \lambda_{1}} p_{\lambda_{1}, \nu_{1}, \mathbf{q}}
$$

where $\epsilon_{\lambda(\nu), \mathbf{k}}^{\mathrm{e}(\mathrm{h})}$ is the electron (hole) single particle energy, $\omega_{\mathrm{R}}=d_{\mathbf{k}}^{\lambda, \nu} E(z, t) / \hbar$ is the Rabi frequency, $d_{\mathbf{k}}^{\lambda, \nu}$ is the dipole matrix element, and $V_{|\mathbf{k}-\mathbf{q}|}^{\lambda, \nu_{1}, \lambda_{1}}$ is the Coulomb potential. The higher order correlation contributions from the Coulomb screening and polarization dephasing $\left(\left.\partial_{t} p_{\lambda, \nu, \mathbf{k}}\right|_{\text {corr }}\right)$ as well as carrier-carrier and carrier-phonon scattering $\left(\left.\partial_{t} n_{\lambda(\nu), \mathbf{k}}^{e(h)}\right|_{\text {corr }}\right)$ can be expressed as multi-dimensional integrals with a nonlinear dependence on the microscopic polarizations and carrier occupation numbers. ${ }^{31}$ In Eq. 2, we also include the carrier recovery from the optical barrier pumping $\left(\Gamma_{\lambda(\nu) \text {,scatt }}^{\mathrm{e}(\mathrm{h})}\right.$ and the spontaneous emissions $\left(\Lambda_{\lambda, \nu, \text { spont }}^{n}\right)$. In addition, we postulate a strong confinement of electrons and holes, which will enable us to simplify Eqs. 2-3. The transition energy for a system with two parabolic energy bands is given by $\hbar \omega_{\mathbf{k}}=E_{\mathrm{g}}+\frac{\hbar^{2} \mathbf{k}^{2}}{2 m_{\mathrm{e}}}+\frac{\hbar^{2} \mathbf{k}^{2}}{2 m_{\mathrm{h}}}$. Where, $m_{\mathrm{e}(\mathbf{h})}$, denotes the effective electron (hole) mass and, $E_{\mathrm{g}}$, the band gap energy.

Numerical evaluation of the high-dimensional integrals, representing the correlation contributions, are in general computationally demanding and hard to directly use for mode-locking simulations. However, assuming a higher QW carrier density one can make an effective rate approximation that significantly speeds up computations. The behavior of the microscopic carrier dynamics can be approximated using a rate that has been found to work well in the past. ${ }^{22}$ The effective rates can be extracted from microscopic QW simulations at the level of second Born-Markov using single pass simulations. The rates contribute to faster simulation times, but will naturally not capture the full range of microscopic dynamics. Nonetheless, the resulting mode-locked pulse found by using these rates is usually close to simulations on the level of second Born-Markov. Thus, these rates give valuable insight on the influence of microscopic dynamics on the propagating electric field.

The effective rates that are extracted from the higher order correlation contributions are: the polarization dephasing $\left(\tau_{\text {deph }}\right)$, the carrier scattering induced kinetic hole filling $\left(\tau_{\text {fill }}\right)$, and the pump injection from barrier scattering $\left(\tau_{\text {scatt }}\right)$. Under rate approximations the specific models are as follows: The polarization dephasing causes a relaxation towards zero with $\left.\partial_{t} p_{\lambda, \nu, \mathbf{k}}\right|_{\text {corr }}=-p_{\lambda, \nu, \mathbf{k}} / \tau_{\text {deph }}$. The carrier scattering equilibriates toward a local equilibrium distribution with $\left.\partial_{t} n_{\lambda(\nu), \mathbf{k}}^{e(h)}\right|_{\text {corr }}=-\left(n_{\lambda(\nu), \mathbf{k}}^{\mathrm{e}(\mathrm{h})}-F_{\lambda(\nu), \mathbf{k}}^{\mathrm{e}}(\mathrm{h}) / \tau_{\text {fill }}\right.$ where $F_{\lambda(\nu), \mathbf{k}}^{\mathrm{e}(\mathrm{h})}$ is a quasi-Fermi distribution at the instantaneous temperature and density. The carrier replenishing from the optical pumping is modeled as a relaxation toward background Fermi distributions, $f_{\lambda(\nu), \mathbf{k}}^{\mathrm{e}(\mathrm{h})}$ at a constant background density and lattice temperature, with $\Gamma_{\lambda(\nu) \text {,scatt }}^{\mathrm{e}(\mathrm{h})}=-\left(n_{\lambda(\nu), \mathbf{k}}^{\mathrm{e}(\mathrm{h})}-f_{\lambda(\nu), \mathbf{k}}^{\mathrm{e}(\mathrm{h})}\right) / \tau_{\text {scatt }}$. The spontaneous emission contributions initialize the electromagnetic field in the cavity and are modeled using $\Lambda_{\lambda, \nu, \text { spont }}^{n}=-\Lambda_{\mathbf{k}}^{\text {spont }} n_{\lambda, \mathbf{k}}^{e} n_{\nu, \mathbf{k}}^{h}$ and $\Lambda_{\lambda, \nu, \text { spont }}^{p}=\beta \Lambda_{\mathbf{k}}^{\text {spont }} n_{\lambda, \mathbf{k}}^{e} n_{\nu, \mathbf{k}}^{h},{ }^{32}$ with parameter $\Lambda_{\mathbf{k}}^{\text {spont }}=\frac{n^{3}}{\pi^{2} \epsilon \hbar^{4} c_{0}^{3}}\left|d_{\mathbf{k}}^{\lambda, \nu}\right|^{2}\left(E_{g}+\frac{\hbar^{2} \mathbf{k}^{2}}{2 \mathrm{~m}_{r}}\right)^{3}$.

A detailed investigation of the microscopic carrier dynamics on the level of second Born-Markov show that carrier scattering can be split into multiple terms. ${ }^{31}$ First, in carrier-carrier scattering, two electrons scatter into unoccupied states under energy- and momentum conservation. The same applies to interactions between holes or with mixtures of particles. Carrier-carrier scattering has a fast characteristic timescale that is on the order of a hundred femtoseconds, as seen in previous studies. ${ }^{22}$ Secondly, in carrier-phonon scattering an electron (or hole) is scattered off the lattice vibrations. This process leads to the cooling of the instantaneous carrier temperature by transporting carriers into lower momentum states. The carrier-phonon scattering has a longer characteristic timescale (of several hundreds femtoseconds ${ }^{33}$ ) and is thus less influential on ultrashort mode-locked pulses, which have a much shorter interaction time with the semiconductor QW medium. However, for single- or multiwavelength operation the electric field is continuously interacting with the QWs. Thus both carrier-carrier and carrier-phonon scattering become important components that will influence the final stable operation of the VECSEL. In this paper, we will include carrier-carrier scattering on the level of second Born-Markov or as an effective rate. This is a first study of multi-mode operation and will later be expanded by including all carrier scattering on the level of second Born-Markov. 


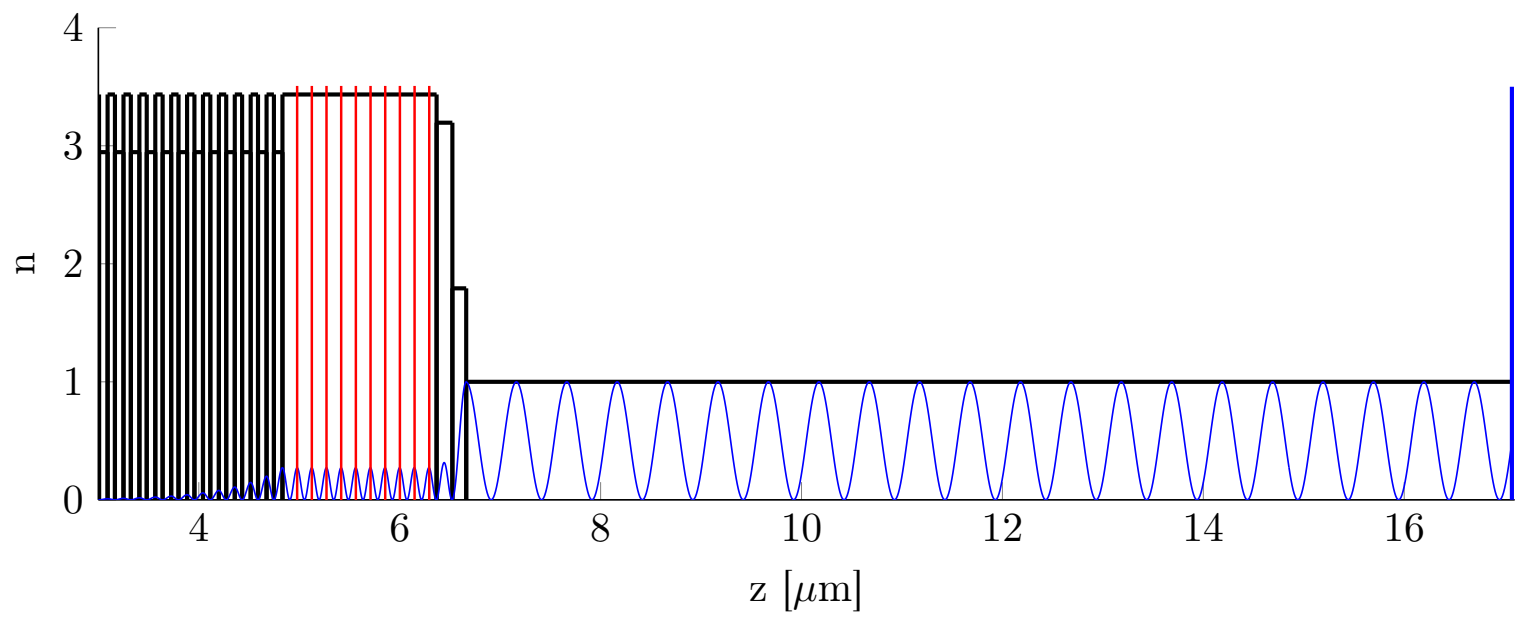

Figure 1: A schematic representation of the simulation domain: The horizontal black bars indicate the refractive index of each material layer; The red vertical bars indicate the location of the optically active QWs; and the blue curve represents the standing wave at $980 \mathrm{~nm}$; The vertical blue bar on the right represents the output coupling mirror.

The simulation domain will be modeled as a linear VECSEL with a gain chip, an air gap, and an output coupler, as illustrated in Fig. 1(a). The gain chip is constructed with an AlGaAs DBR, ten inverted $8 \mathrm{~nm}$ wide InGaAs QWs in a resonant periodic placement, a cap layer, and a dispersion compensating coating. Similar to designs that have been used to produce high CW output power. ${ }^{6,7}$ In our simulations the DBR and peak gain are both centered at the operating wavelength $980 \mathrm{~nm}$. This design has a single layer of dispersion compensating coating, but a coating can have multiple layers where the lengths are numerically optimized for low total dispersion. When initializing mode-locking simulations the QW background carrier density is constant, at values given in the text below, with a background temperature at the lattice temperature $(300 \mathrm{~K})$.

\section{RESULTS}

A VECSEL can be configured to produce ultrashort mode-locked pulses (by including a saturable absorber) or high power single- or multi-mode output (with only an output coupling mirror). The resulting high-intensity intracavity fields or wide spectrum mode-locked pulses produce highly non-equilibrium QW carrier distributions. In the optically active QWs, the electromagnetic field extracts QW carriers simultaneously as carrier scattering equilibrates carriers to quasi-Fermi distributions. The stability and ultimate limits of the VECSEL output is determined by the microscopic QW dynamics. It is useful to study the inversion, $n_{\lambda, \mathbf{k}}^{\mathrm{e}}+n_{\nu, \mathbf{k}}^{\mathrm{h}}-1$, in the context of pulsed operation, to understand how the microscopic dynamics influences the intracavity field.

The microscopic QW carrier inversion enters directly into Eq. 2 where inverted carriers will result in spectral amplification. For a given gain chip, the shape of the gain spectrum will depend on the specific state of QW carriers as well as the gain chip architecture. The simplest method used to calculate the spectral amplification from a gain chip with multiple QWs is to use a single pass of a low-energy probe pulse. Here, we compare the pulse spectrum before and after interacting with the gain chip. However, it is not possible to invert this problem without further assumptions (i.e. find the carrier distribution that produces a given gain curvature). Fig. 2 shows a simple example that illustrates how multiple different non-equilibrium carrier distributions can produce very similar gain curves. In this case, the gain curves seen in Fig. 2(a) are calculated from the corresponding carrier distributions in Fig. 2(b). In order to generate this example, we hand pick a couple of carrier distributions that produce nearly the same gain curve. The QW carrier density that gives the dashed line is $1.9 \cdot 10^{16} \mathrm{~m}^{-2}$ and the carrier density of the other two lines are approximately $2.0 \cdot 10^{16} \mathrm{~m}^{-2}$. From this example, we see that it is difficult to draw conclusions about microscopic dynamics based on the optical gain and thus what influence the QW carriers have on the propagating electromagnetic field. 

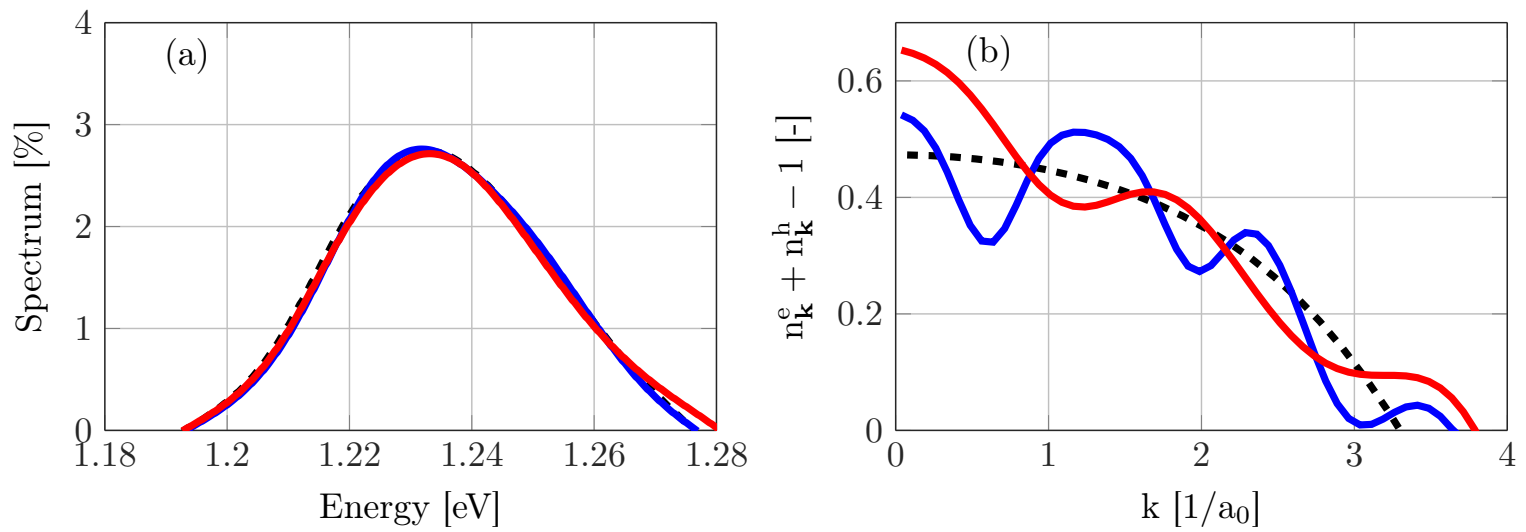

Figure 2: A comparison of the computed optical gain (a) from the corresponding microscopic QW carrier inversion (b), from the gain chip shown in Fig. 1(a).

The dynamical details, such as pulse shapes and intensities, under stable mode-locking conditions can be characterized by the QW background inversion and by how many carriers the pulse extracts during a round trip. $^{23}$ If the inversion is reduced to zero at some momentum value we say that the inversion is bleached i.e. there in no amplification or absorption from this momentum value. For a low level of background inversion the mode-locked pulse never grows strong enough to bleach the instantaneous inversion and a single stable mode-locked pulse can be found in the cavity. For a medium level of background inversion, the pulse grows strong enough to extract all inverted carriers at some momentum value. In this case, a higher pulse intensity will result in absorption from the bleached momentum values. However, the pulse does not have to break up completely. Instead, the pulse spectrum tries to extract more energy by reshaping into two spectral peaks and forms a so-called pulse molecule. This temporal shape, typically corresponding to a double peaked spectrum and is recognized as a series of short pulses inside a longer envelope. In general, this pulse has some substructure that is changing over multiple round-trips and is not a stable mode-locked pulse. For a high level of background inversion it is not possible for a single pulse to extract all carriers in a single round-trip. Thus, unsaturated carriers will form additional pulses and causes multiple pulses to appear in the cavity. In this situation, it is not possible to find a stable mode-locked pulse without first reducing the QW background carrier density.
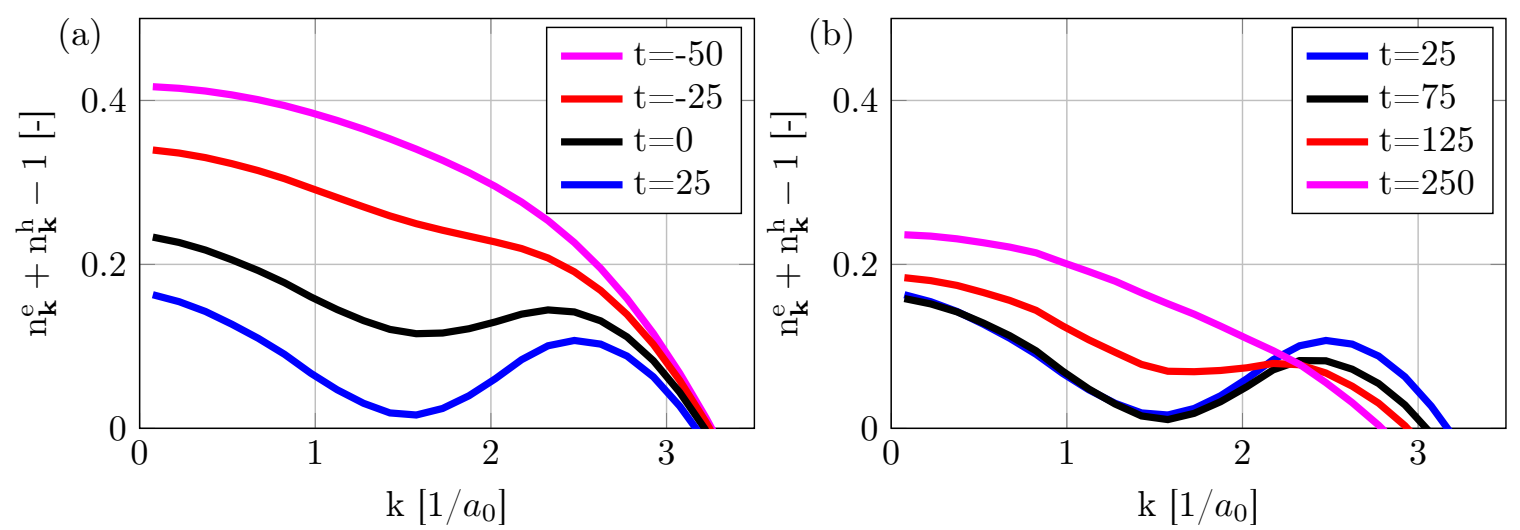

Figure 3: A simulation where only $\left.\frac{\partial}{\partial t} n_{\lambda(\nu), \mathbf{k}}^{e(h)}\right|_{\text {corr }}$ is used to redistribute carriers during interaction with a pulse where the legend indicates the number of femtoseconds relative to the pulse center. (a) The pulse is extracting carriers from a QW. (b) Carrier scattering is redistributing carriers.

High intensity intracavity fields will drive the microscopic QW distributions away from equilibrium and carrier scattering $\left(\left.\frac{\partial}{\partial t} n_{\lambda(\nu), \mathbf{k}}^{e(h)}\right|_{\text {corr }}\right)$ drives non-equilibrium distributions towards quasi-Fermi distributions. To illustrate this feature, we show in Fig. 3(a) snapshots of the inversion, at select times relative to the pulse peak, as a 
pulse extracts carriers from an inverted QW. In this figure, pump recovery and spontaneous emissions have been disabled to demonstrate the role of carrier scattering. The removed carriers leave behind a kinetic hole in the inversion with the shape of the pulse spectrum. If the pulse has a high intensity then the inversion could become bleached. Fig. 3(b) shows the QW inversion after the pulse has left. Here, we can see how the high momentum carriers are scattered down into the lower momentum states. This process is simply referred to as kinetic hole filling.

These simulations are started from spontaneous emission noise, which allows us to establish the final stable field by iterating over multiple round-trips. The initial low-energy noise will be amplified by the gain chip and experience loss from the output coupling mirror. There will be some spectral range that experiences a positive net round-trip amplification (i.e. the net gain region). Eventually, the cavity field will be strong enough to extract a significant number of carriers from the gain chip and thus reduce the carrier density. The growing spectral amplitude of the cavity field will stop once the gain chip amplification is equal to the output coupling loss. The length of the external cavity will restrict the available cavity modes that constructively amplify from noise. A short cavity will create a wide mode spacing and reduces the computational time required to obtain a stable output. This enables us to run simulations with carrier scattering at the level of second Born-Markov for the entire simulation. A long external cavity will decrease the cavity mode spacing and increase the computational cost of each round-trip. In this case, carrier scattering is calculated using an approximate kinetic hole filling rate.
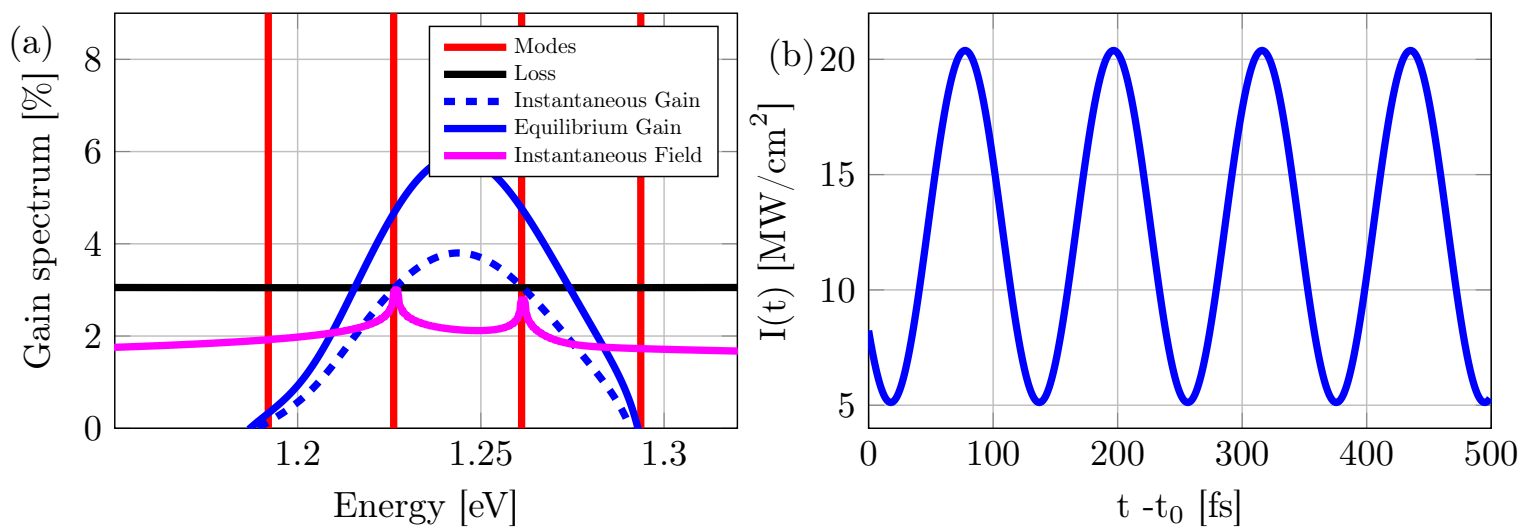

Figure 4: The stable dual-wavelength solution from a short external cavity with round-trip time around $0.2 \mathrm{ps}$, a QW background carrier density $5.0 \cdot 10^{16} \mathrm{~m}^{-2}$, output coupling loss $3 \%$, and a relaxation time $\tau_{\text {scatt }}=1 \mathrm{ps}$. (a) The cavity modes (red), the output loss (black), initial gain (solid blue), the instantaneous gain during stable operation (dashed blue), and the logarithm of the output field intensity (magenta). (b) A time trace of the cavity field intensity at a QW.

In Fig. 4, we can see the final dual-wavelength output from a simulation using the gain chip shown in Fig. 1. The final output field has spectral peaks at $1.227 \mathrm{eV}$ and $1.262 \mathrm{eV}$, close to the cavity modes shown in Fig. 4(a). We note that the frequencies might not perfectly overlap with the cavity modes due to frequency pulling by the optically active QWs. From the cavity field spectrum, we observe that there is no significant noise in the signal, but the spectral amplitudes of the two modes are not equal. The uneven spectral amplitudes are also reflected in the output field intensity, seen in Fig. 4(b). Here, the output field is oscillating with the beating frequency of the two spectral peaks.

The final non-equilibrium cavity field has established a balance between the gain chip QW amplification and the output coupling loss under the constraints of the VECSEL cavity. Fig. 5(a) gives a snapshot of the QW state during stable operation and it is clear that the two cavity modes burn kinetic holes in the QW carrier distributions with different depths. The oscillation pattern of the beating cavity field is also present in the kinetic holes. Fig. 5(b) shows how the carrier inversion changes over time. The two spectral peaks from the cavity field burn individual kinetic holes into the carrier distributions and the carrier inversion is oscillating with the beating frequency given by the two spectral peaks. 

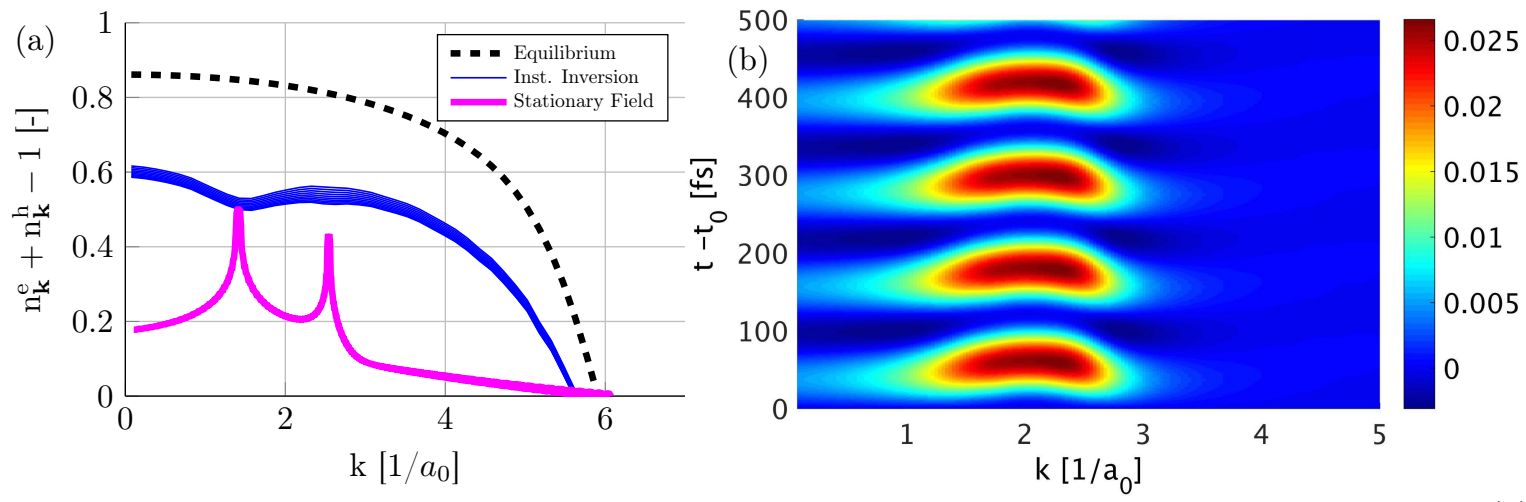

Figure 5: A snapshot of the QW carriers during stable dual-wavelength operation shown in Fig. 4. (a) The background carrier inversion (dashed black), the instantaneous carrier inversion of all QWs (solid blue), and the logarithm of the output field. (b) The change in inversion of a QW during stable operation.

Complex non-equilibrium dynamics is present on many timescales in the dual-wavelength output seen in Fig. 4. The two frequencies create an oscillating field intensity, which is also present as carrier oscillations in the QWs. The final output is also slowly changing over many round-trips, and this is intimately connected to the carrier density of the gain chip QWs. Fig. 6(a) shows how the peak spectral intensity of the two wavelengths change over thousands of round-trips. Here, it is clear that the amplitudes of both frequencies oscillate in phase, but out of phase with the changing QW carrier density, shown in Fig. 6(b), as is typical for are gain relaxation oscillations.
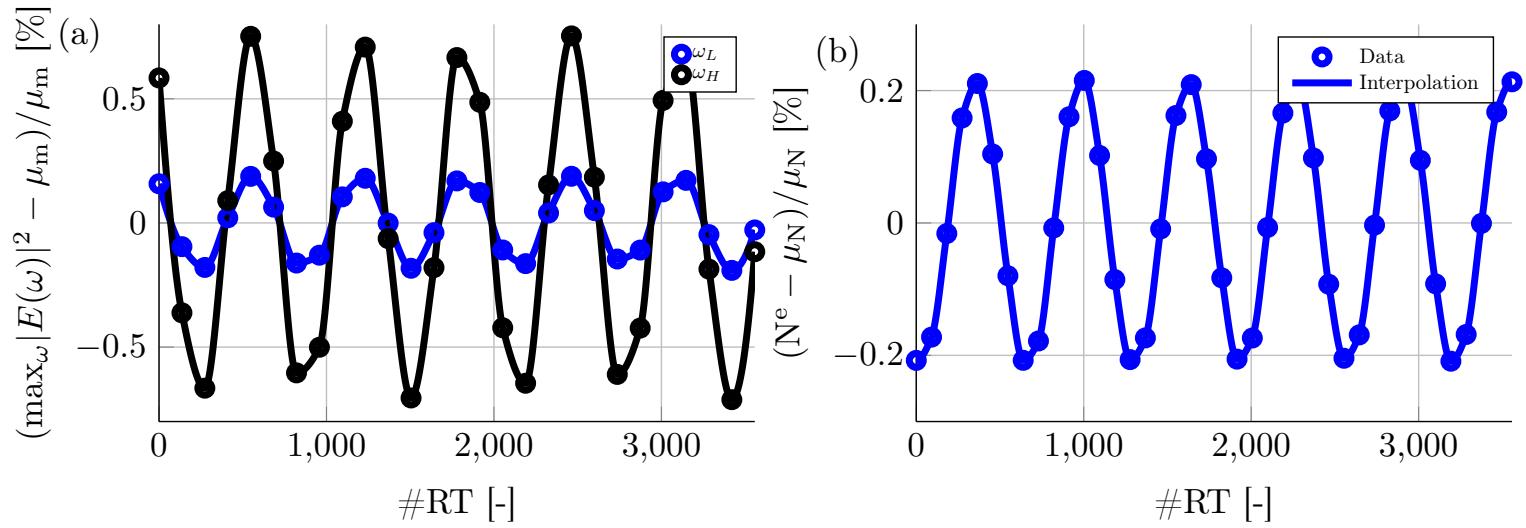

Figure 6: The change in dual-wavelength output from Figs. 4-5 over many round-trips of stable operation. The solid lines are interpolations to guide the eye and the dots are sampled data. (a) The relative change in peak intensity of each spectral peak $\left(\omega_{L}=1.227 \mathrm{eV}\right.$ and $\left.\omega_{H}=1.262 \mathrm{eV}\right)$. (b) The relative change in QW carrier density of a QW.

In contrast to single-wavelength operation, in dual-wavelength output the $\mathrm{QW}$ carriers have established a non-equilibrium state that supports the beating electromagnetic field. In the stable asymptotic cavity field, carrier replenishing and carrier scattering are both transporting carriers into lower momentum states. For our example, we set the carrier replenishing time $\left(\tau_{\text {scatt }}\right)$ to $1 \mathrm{ps}$ because this simulation does not include carrierphonon scattering, which will scatter more carriers into the lower momentum range. Another important aspect is that the frequency selectivity of the short cavity results in two very narrow cavity modes. A dual-wavelength solution has to balance all the microscopic dynamics with the output coupling loss and the constraints from the cavity modes. Thus, a longer cavity should give the system more flexibility in establishing dual-wavelength operation.

The number of cavity modes increases significantly when the external cavity round-trip time is increased to 

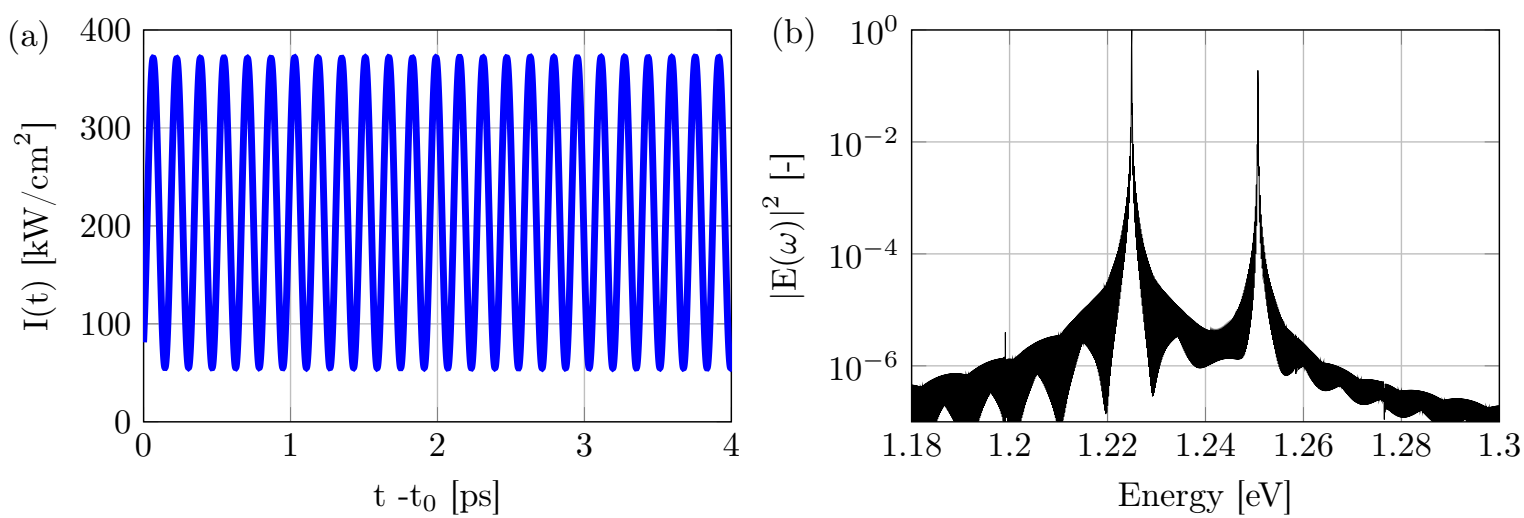

Figure 7: A stable dual-wavelength solution from a long external cavity with a 22 ps round-trip time, two etalons to select frequencies, a QW background carrier density $1.9 \cdot 10^{16} \mathrm{~m}^{-2}$, output coupling loss $1 \%$, and a relaxation time $\tau_{\text {scatt }}=0.2$ ps. (a) The output field intensity. (b) The spectrum of the output field.

22 ps. In order to increase the frequency selectivity of the VECSEL, we include a pair of glass etalons (refractive indices 1.5) of length $98 \mu \mathrm{m}$ and $5 \mu \mathrm{m}$ into the cavity. The longer cavity also increases the simulation time required to obtain a stable solution and thus we use the rate approximation to kinetic hole filling in order to generate results within a realistic timeframe. Fig. 7(a) shows the final dual-wavelength output intensity and in (b) we can see the spectrum. The two spectral peaks are located at $1.225 \mathrm{eV}$ and $1.251 \mathrm{eV}$ that results in a separation of about $6 \mathrm{THz}$. Note that in Fig. 7(b) it is possible to see the signature of four-wave-mixing at the spectral peak near $1.2 \mathrm{eV}$.

In Figs. 4-7 the carrier replenishing time is set at a relatively low value. This is in order to ensure that enough carriers are scattered into the lower momentum states, in the absence of carrier-phonon scattering. However, the results are representative for the type of behavior one expects to see in these systems.

\section{SUMMARY}

A numerical simulation is presented of a VECSEL that includes the microscopic non-equilibrium dynamics using the Maxwell semiconductor Bloch equations. We examine the role of the QW gain versus the QW inversion in establishing a mode-locked pulse and dual-wavelength output. A stable dual-wavelength output is an asymptotic non-equilibrium solution to the MSBE, which couples the microscopic dynamics with the external cavity setup including cavity modes and output coupling loss. We find that there is ultrafast carrier dynamics on many timescales during stable dual-wavelength operation. Thus, in order to accurately model dual-wavelength output we need a model that includes microscopic QW dynamics with carrier replenishing, carrier-carrier, and carrierphonon scattering. The carrier-phonon scattering is essential in transporting carriers into the lower momentum states and thus increasing the available carriers for field amplification. In addition, we find that the dualwavelength output is sensitive to the mode setup of the cavity.

\section{ACKNOWLEDGEMENTS}

This material is based upon work supported by the Air Force Office of Scientific Research under award number FA9550-14-1-0062 and FA9550-17-1-0246.

\section{REFERENCES}

[1] Garnache, A., Hoogland, S., Tropper, A. C., Sagnes, I., Saint-Girons, G., and Roberts, J. S., "Sub-500-fs soliton-like pulse in a passively mode-locked broadband surface-emitting laser with $100 \mathrm{~mW}$ average power," Applied Physics Letters 80(21), 3892-3894 (2002).

[2] Keller, U. and Tropper, A. C., "Passively modelocked surface-emitting semiconductor lasers," Phys. Rep. 429, 67-120 (2006). 
[3] Quarterman, A. H., Wilcox, K. G., Apostolopoulos, V., Mihoubi, Z., Elsmere, S. P., Farrer, I., Ritchie, D. A., and Tropper, A., "A passively mode-locked external-cavity semiconductor laser emitting 60-fs pulses," Nat. Photonics 3, 729-731 (2009).

[4] Hoffmann, M., Sieber, O. D., Wittwer, V. J., Krestnikov, I. L., Livshits, D. A., Barbarin, Y., Südmeyer, T., and Keller, U., "Femtosecond high-power quantum dot vertical external cavity surface emitting laser," Opt. Express 19, 8108-8116 (2011).

[5] Tropper, A. C., Quarterman, A. H., and Wilcox, K. G., "Ultrafast vertical-external-cavity surface-emitting semiconductor lasers," Advances in Semiconductor Lasers 86, 269-300 (2012).

[6] Heinen, B., Wang, T.-L., Sparenberg, M., Weber, A., Kunert, B., Hader, J., Koch, S. W., Moloney, J. V., Koch, M., and Stolz, W., "106 W continuous-wave output power from vertical-external-cavity surfaceemitting laser," Electron. Lett. 48(9), 516 (2012).

[7] Wang, T.-L., Heinen, B., Hader, J., Dineen, C., Sparenberg, M., Weber, A., Kunert, B., Koch, S. W., Moloney, J. V., Koch, M., and Stolz, W., "Quantum design strategy pushes high-power vertical-externalcavity surface-emitting lasers beyond $100 \mathrm{~W}$," Laser Photon. Rev. 6(5), L12-L14 (2012).

[8] Scheller, M., Wang, T.-L., Kunert, B., Stolz, W., Koch, S. W., and Moloney, J. V., "Passively modelocked vecsel emitting $682 \mathrm{fs}$ pulses with $5.1 \mathrm{~W}$ of average output power," Electron. Lett. 48(10), 588-589 (2012).

[9] Wilcox, K. G., Tropper, A. C., Beere, H. E., Ritchie, D. A., Kunert, B., Heinen, B., and Stolz, W., "4.35 kW peak power femtosecond pulse mode-locked vecsel for supercontinuum generation," Opt. Express 21(2), 1599-1605 (2013).

[10] Zaugg, C., Sun, Z., Wittwer, V. J., Popa, D., Milana, S., Kulmala, T. S., Sundaram, R. S., Mangold, M., Sieber, O. D., Golling, M., Lee, Y., Ahn, J. H., Ferrari, A. C., and Keller, U., "Ultrafast and widely tuneable vertical-external-cavity surface-emitting laser, mode-locked by a graphene-integrated distributed bragg reflector," Opt. Express 21, 31548-31559 (2013).

[11] Husaini, S. and Bedford, R. A., "Antiresonant graphene saturable absorber mirror for mode-locking VECSELs." personal communication (2013).

[12] Seger, K., Meiser, N., Choi, S. Y., Jung, B. H., Yeom, D.-I., Rotermund, F., Okhotnikov, O., Laurell, F., and Pasiskevicius, V., "Carbon nanotube mode-locked optically-pumped semiconductor disk laser," Optics express 21(15), 17806-17813 (2013).

[13] Klopp, P., Griebner, U., Zorn, M., and Weyers, M., "Pulse repetition rate up to $92 \mathrm{GHz}$ or pulse duration shorter than 110 fs from a mode-locked semiconductor disk laser," Appl. Phys. Lett. 98, 071103 (2011).

[14] Zhang, F., Heinen, B., Wichmann, M., Möller, C., Kunert, B., Rahimi-Iman, A., Stolz, W., and Koch, M., "A 23-watt single-frequency vertical-external-cavity surface-emitting laser," Optics express 22(11), 12817-12822 (2014).

[15] Laurain, A., Mart, C., Hader, J., Moloney, J. V., Kunert, B., and Stolz, W., "15 W single frequency optically pumped semiconductor laser with sub-megahertz linewidth," IEEE Photonics Technology Letters 26(2), 131$133(2014)$.

[16] Waldburger, D., Link, S. M., Mangold, M., Alfieri, C. G. E., Gini, E., Golling, M., Tilma, B. W., and Keller, U., "High-power 100 fs semiconductor disk lasers," Optica 3(8), 844-852 (2016).

[17] Alfieri, C. G. E., Waldburger, D., Link, S. M., Gini, E., Golling, M., Eisenstein, G., and Keller, U., "Optical efficiency and gain dynamics of modelocked semiconductor disk lasers," Optics Express 25(6), 6402-6420 (2017).

[18] Sieber, O. D., Hoffmann, M., Wittwer, V. J., Mangold, M., Golling, M., Tilma, B. W., Südmeyer, T., and Keller, U., "Experimentally verified pulse formation model for high-power femtosecond vecsels," Applied Physics B 113(1), 133-145 (2013).

[19] Paschotta, R., "Noise of mode-locked lasers (part i): numerical model," Applied Physics B: Lasers and Optics 79(2), 153-162 (2004).

[20] Haug, H. and Koch, S. W., [Quantum Theory of the Optical and Electronic Properties of Semiconductors], World Scientific, Singapore, $5^{\text {th }}$ ed. (2009).

[21] Kilen, I., Koch, S. W., Hader, J., and Moloney, J. V., "Non-equilibrium ultrashort pulse generation strategies in vecsels," Optica 4(4), 412-417 (2017). 
[22] Kilen, I., Koch, S. W., Hader, J., and Moloney, J. V., "Fully microscopic modeling of mode locking in microcavity lasers," JOSA B 33(1), 75-80 (2016).

[23] Kilen, I., Hader, J., Moloney, J. V., and Koch, S. W., "Ultrafast nonequilibrium carrier dynamics in semiconductor laser mode locking," Optica 1(4), 192-197 (2014).

[24] Hader, J., Scheller, M., Laurain, A., Kilen, I., Baker, C., Moloney, J. V., and Koch, S. W., "Ultrafast non-equilibrium carrier dynamics in semiconductor laser mode-locking," Semiconductor Science and Technology 32(1), 013002 (2016).

[25] Moloney, J. V., Kilen, I., Bäumner, A., Scheller, M., and Koch, S. W., "Nonequilibrium and thermal effects in mode-locked vecsels," Optics express 22(6), 6422-6427 (2014).

[26] Böttge, C. N., Hader, J., Kilen, I., Moloney, J. V., and Koch, S. W., "Ultrafast pulse amplification in modelocked vertical external-cavity surface-emitting lasers," Applied Physics Letters 105(26), 261105 (2014).

[27] Hader, J., Kilen, I., Moloney, J. V., and Koch, S. W., "Non-equilibrium effects in vecsels," in [Proc. of SPIE Vol], 10087, 1008706-1 (2017).

[28] Kilen, I., Koch, S. W., Hader, J., and Moloney, J. V., "Modeling of ultrashort pulse generation in modelocked vecsels," in [SPIE OPTO], 97420H-97420H, International Society for Optics and Photonics (2016).

[29] Kilen, I., Böttge, C. N., Hader, J., Koch, S. W., and Moloney, J. V., "Ultrafast non-equilibrium carrier dynamics in semiconductor laser mode-locking," in [SPIE OPTO], 934902-934902, International Society for Optics and Photonics (2015).

[30] Böttge, C. N., Hader, J., Kilen, I., Koch, S. W., and Moloney, J. V., "Influence of non-equilibrium carrier dynamics on pulse amplification in semiconductor gain media," in [SPIE LASE], 934903-934903, International Society for Optics and Photonics (2015).

[31] Hader, J., Koch, S. W., and Moloney, J. V., "Microscopic theory of gain and spontaneous emission in gainnas laser material," Solid-State Electronics 47(3), 513-521 (2003).

[32] Bäumner, A., Koch, S. W., and Moloney, J. V., "Non-equilibrium analysis of the two-color operation in semiconductor quantum-well lasers," physica status solidi (b) 248(4), 843-846 (2011).

[33] Chow, W. W. and Koch, S. W., [Semiconductor-laser fundamentals: physics of the gain materials], Springer Science \& Business Media (2013). 